\title{
Evolution in Sinocyclocheilus cavefish is marked by rate shifts, reversals, and origin of novel traits
}

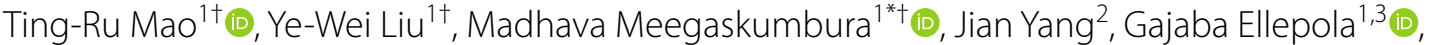 \\ Gayani Senevirathne ${ }^{4}$, Cheng-Hai Fu', Joshua B. Gross ${ }^{5}$ (D) and Marcio R. Pie ${ }^{6}$ (D)
}

\begin{abstract}
Background: Natural model systems are indispensable for exploring adaptations in response to environmental pressures. Sinocyclocheilus of China, the most diverse cavefish clade in the world (75 species), provide unique opportunities to understand recurrent evolution of stereotypic traits (such as eye loss and sensory expansion) in the context of a deep and diverse phylogenetic group. However, they remain poorly understood in terms of their morphological evolution. Therefore, we explore key patterns of morphological evolution, habitat utilization and geographic distribution in these fishes.
\end{abstract}

Results: We constructed phylogenies and categorized 49 species based on eye-related condition (Blind, Micro-eyed, and Normal-eyed), habitat types (Troglobitic — cave-restricted; Troglophilic_cave-associated; Surface—outside caves) and existence of horns. Geometric-morphometric analyses show Normal-eyed morphs with fusiform shapes segregating from Blind/Micro-eyed deeper bodied morphs along the first principal-component axis; second axis accounts for shape complexity related to horns. The body shapes showed a significant association with eye-related condition and horn, but not habitat types. Ancestral reconstructions suggest at least three independent origins of Blind morphs, each with different levels of modification in relation to their ancestral Normal-eyed morphs; Sinocyclocheilus are also pre-adapted for cave dwelling. Our geophylogeny shows an east-to-west diversification spanning Pliocene and Pleistocene, with early-diversifying Troglobitic species dominating subterranean habitats of karstic plains whereas predominantly Surface forms inhabit hills to the west. Evolutionary rates analyses suggest that lineages leading to Blind morphs were characterized by significant rate shifts, such as a slowdown in body size evolution and a 5-20 fold increase in rate of eye regression, possibly explained by limited resource availability. Body size and eye size have undergone reversals, but not horns, a trait entailing considerable time to form.

Conclusions: Sinocyclocheilus occupied cave habitats in response to drying associated with aridification of China during late Miocene and the Pliocene. The prominent cave-adaptations (eye-regression, horn-evolution) occur in clades associated with the extensive subterranean cave system in Guangxi and Guizhou provinces. Integration of morphology, phylogeny, rate analyses, molecular-dating and distribution show not only several remarkable patterns

\footnotetext{
*Correspondence: madhava_m@mac.com

†Ting-Ru Mao, Ye-Wei Liu and Madhava Meegaskumbura have equal contributions

${ }^{1}$ Guangxi Key Laboratory for Forest Ecology and Conservation, College

of Forestry, Guangxi University, Nanning, Guangxi, People's Republic

of China

Full list of author information is available at the end of the article
}

(C) The Author(s) 2021. Open Access This article is licensed under a Creative Commons Attribution 4.0 International License, which permits use, sharing, adaptation, distribution and reproduction in any medium or format, as long as you give appropriate credit to the original author(s) and the source, provide a link to the Creative Commons licence, and indicate if changes were made. The images or other third party material in this article are included in the article's Creative Commons licence, unless indicated otherwise in a credit line to the material. If material is not included in the article's Creative Commons licence and your intended use is not permitted by statutory regulation or exceeds the permitted use, you will need to obtain permission directly from the copyright holder. To view a copy of this licence, visit http://creativecommons.org/licenses/by/4.0/. The Creative Commons Public Domain Dedication waiver (http://creativeco mmons.org/publicdomain/zero/1.0/) applies to the data made available in this article, unless otherwise stated in a credit line to the data. 
of evolution, but also interesting exceptions to these patterns signifying the diversification of Sinocyclocheilus as an invaluable model system to explore evolutionary novelty.

Keywords: Phylomorphospace, Evolutionary convergence, Blind fish, Troglobitic, Geophylogeny

\section{Background}

Due to the absence of light, absence of primary productivity, frigid temperatures and paucity of dissolved oxygen, subterranean habitats are among some of the most challenging environments for life on earth $[1,2]$. From surface-dwelling ancestral species, cavefish have secondarily adapted to live in cave systems, often demonstrating a remarkable array of morphological and behavioral adaptations [3, 4]. These involve enhanced sensation, and also dispensing of traits that incur a developmental or energetic cost. Cavefish species can be divided into two forms-Troglophilic closely associated with caves, but not entirely dependent on them, and Troglobitic, the obligate cave dwellers $[5,6]$. Troglobitic species may bear special adaptations, such as complete eye loss, loss of pigmentation, changes in cranial symmetry, proliferation of neuromast sensory organs, development of horns, and in some species flat, hollow heads [7-9]. Despite the $\sim 200$ cavefish species being known from across the world, large diversifications of cavefishes are rare. However, one extensive diversification occurs in Sinocyclocheilus (Cyprinidae, Barbinae), a monophyletic group of cyprinid fishes endemic to China, which allows a robust analysis of trait evolution relative to troglomorphism in a phylogenetic context.

The specialized traits cavefish bear have led them to be investigated as models of evolution, especially with respect to adaptations to novel environments and evolutionary convergence [5, 7, 10-15]. A lion's share of knowledge on evolution and development in cavefishes has come from Astyanax mexicanus (Mexican tetra), a species with both surface-dwelling (pigmented and eyed) and cave-dwelling morphs (depigmented and blind), which readily interbreed [16]. In contrast to this wellstudied model, Sinocyclocheilus species not only include blind and normal-eyed morphs [17], but demonstrate a continuum from blind to normal-eyed species. Indeed, members of the Sinocyclocheilus genus display remarkable morphological evolution with divergent cave-dwelling, cave-associated, and surface-dwelling species.

Sinocyclocheilus species are thought to have shared a common ancestor in the late Miocene, undergoing a spectacular diversification spanning the Pliocene and Pleistocene, across the southwestern parts of China's $620,000 \mathrm{~km}^{2}$ of karst habitats [18], with nearly 75 extant species [19]. This resulted in an adaptive diversification into subterranean refugia traversing the intersection of the Guizhou, Guangxi and Yunnan provinces around the time of the uplifting of Tibetan/Guizhou plateau [12].

One of the most striking forms of cave adaptation in Sinocyclocheilus is variation in eye morphology, categorized often into three morphs [20], ranging from Normaleyed, through Micro-eyed (small-eyed) to Blind species. Of all Chinese hypogean fishes, 56 species show troglomorphism such as reduction and/or loss of eyes, pigmentation, and the gas bladder. Presence of a horn-like structure and hyper-development of the dorsal protuberance (humped back) are two additional unique characters to certain Chinese hypogean species [21]. These dramatic adaptations to cave life are reflected in the unique morphology of these fish.

While the morphology of Sinocyclocheilus is likely attributed to their habitat and local adaptations, the precise function of certain morphologies (e.g., their horns) remains unknown [22]. For instance, many blind species are obligate cave dwellers that have the ability to navigate along cave walls, cave-bottoms and within narrow passages [4]. Yet, others are open-water species that navigate in the manner typical of fish. There are also intermediate forms between these two principal morphs [20]. However, the morphology of these fishes is so extreme and substantial variation in morphology is evident even within blind, intermediate and open-water species. We chose to examine evolution of shape related variation of these fishes using mainly geometric morphometrics and ancestral state reconstructions.

Much of the work on Sinocyclocheilus has been taxonomic in nature [23], and some species are only known from a few specimens or photographs [23-31], and in some cases, only from a single type specimen [19]. Over the past decade, however, the inventory of Sinocyclocheilus species has steadily increased $[6,19,20,23-25$, 28-39], indicating that there are more species yet to be discovered. Difficulties in sampling deep caves scattered across the karstic expanse, and the extreme rarity of some species have impeded exploratory and monitoring studies. Hence we build on previous data (genetic, morphology and distribution), together with the data that we accumulated over 3 years.

Sinocyclocheilus is an emerging model system and studies have explored gene mapping, eye degeneration, and mechanics of the lateral line system [14, 40-42], albeit in only a few handpicked species from across the phylogeny. However, future studies would benefit from a 


\section{(See figure on next page.)}

Fig. 1 Ancestral character state reconstruction using stochastic character mapping for the eye-trait morphology (Blind, Micro and Normal-eyed morphs) on a time calibrated phylogeny of using a "standard clock" estimate. A, B, C and D are four major clades. Posterior probabilities of node support values of $100 \%$ are indicated by *. Key events of Sinocyclocheilus evolution includes; at least three independent evolutionary events for Blind morphs; Blind, Micro-eyed and a few Normal-eyed species in Clade B, with two cases of reversal from either Micro-eyed or Blind to Normal-eyed species. Sinocyclocheilus diversification seems to have initiated in the mid-Miocene with the aridification of China

comprehensive analysis of the evolution of major traits of the Sinocyclocheilus diversification and would bolster both comparative work and hypothesis testing in this group of fishes.

Here, we explore key patterns of morphological evolution, habitat utilization and geographic distribution in these fishes. We ask the following main questions: What are the patterns of evolution of morphological diversity and habitat utilization? What is the geographic distribution and chronology of this diversity?

We show evolution in Sinocyclocheilus to have been associated with convergence, significant rate changes and trait reversals across their phylogenetic history and that adaptations for cave dwelling originated in the earlyemerging clades inhabiting the eastern expanse of their distribution.

\section{Results}

Phylogenetic inference and ancestral state reconstruction The maximum credibility tree of Sinocyclocheilus is shown in Fig. 1, together with the reconstruction of ancestral states for eye-related morphs; we consider four major clades (A, B, C, D), as previously reported by other authors [20]. Despite the uncertainty inherent in ancestral state reconstructions, it is clear that Blind species evolved at least three times in Sinocyclocheilus. Two of these events involved single species evolving from Normal-eyed ancestors, namely S. xunlensis and S. anophthalmus. On the other hand, the third lineage of blind Sinocyclocheilus, Clade B, includes several closely related species of Blind, Micro-eyed and a few Normal-eyed species, with two cases of reversal from either Micro-eyed or Blind to Normal-eyed morphs, namely S. zhenfengensis and S. brevibarbatus (Fig. 1). All four clades contain Regressed-eyed species and comparatively, Clade B contains most cases with Regressed-eyed species. Our analyses indicate that Clade B originated in the early Miocene about 5.5 million years ago, whereas the other two transitions to blind species were much more recent. Our research result also shows that most recent common ancestor of Sinocyclocheilus originated about 7.4 million years ago (Fig. 1). However, in the approach using two calibration points to estimate divergence time, Clade B seems to have originated about 6 million years ago, and the divergence time between the species in Clade $\mathrm{A}$ and other species in the genus Sinocyclocheilus is about 8.5 million years ago (Additional file 1: Fig. S1).

Ancestral state reconstruction of habitat type indicated (C) that troglobitic ancestral species have evolved first and surface dwellers evolved later, especially the species in Clade D. Few species in clades B and C (S. brevibarbatus, S. multipunctatus, S. macrolepis and S. longibarbatus) show reversals from troglobitic to troglophilic recently in their evolution whereas species in Clade D evolved to predominantly a surface habitats since the Pliocene. Further, S. anophthalmus in Clade D exhibit a second event of cave origin fairly recently in the phylogeny.

In our study, horned morphs have originated from a non-horned ancestor and are restricted to Clade B where they exhibit a pattern such that sister taxa of a horned morph is a non-horned species in most cases (Additional file 1: Fig. S2).

\section{Patterns of morphological trait evolution and phylomorphospace analysis}

Our analyses shows that the evolution of body size and eye diameter in Sinocyclocheilus showed rather distinct dynamics (Fig. 2). For instance, body size evolved at a relatively constant rate early in the evolution of the genus, with an apparently accelerated rate near the present, but with little correspondence with eye morphs. Indeed, normal-eyed species span the entire range of body sizes (Fig. 2a). On the other hand, there was an early differentiation in eye diameter at around 4.7 Mya between normal and micro-eyed/blind species, but many other reversals took place near the present (Fig. 2b, c). These patterns suggest that eye and body size evolution in Sinocyclocheilus were largely decoupled during its history. Habitat associations traced on phylomorphospace (Fig. 2d) indicate that species with regressed eyes and smallto-medium body sizes are obligate cave dwellers. This same pattern is shown by the standardized eye diameter related trigrams also (Additional file 1: Fig. S3). However, normal eyed species can be Troglobitic, Troglophilic or surface dwellers regardless of their body size. Our results indicate that all horned species are obligate cave dwellers while not all cave species are horned (Fig. 2e).

A more precise description of the overall changes associated with different morphs can be visualized in the projections built from the geometric morphometrics analyses (Fig. 3b). The first PC, which accounted 


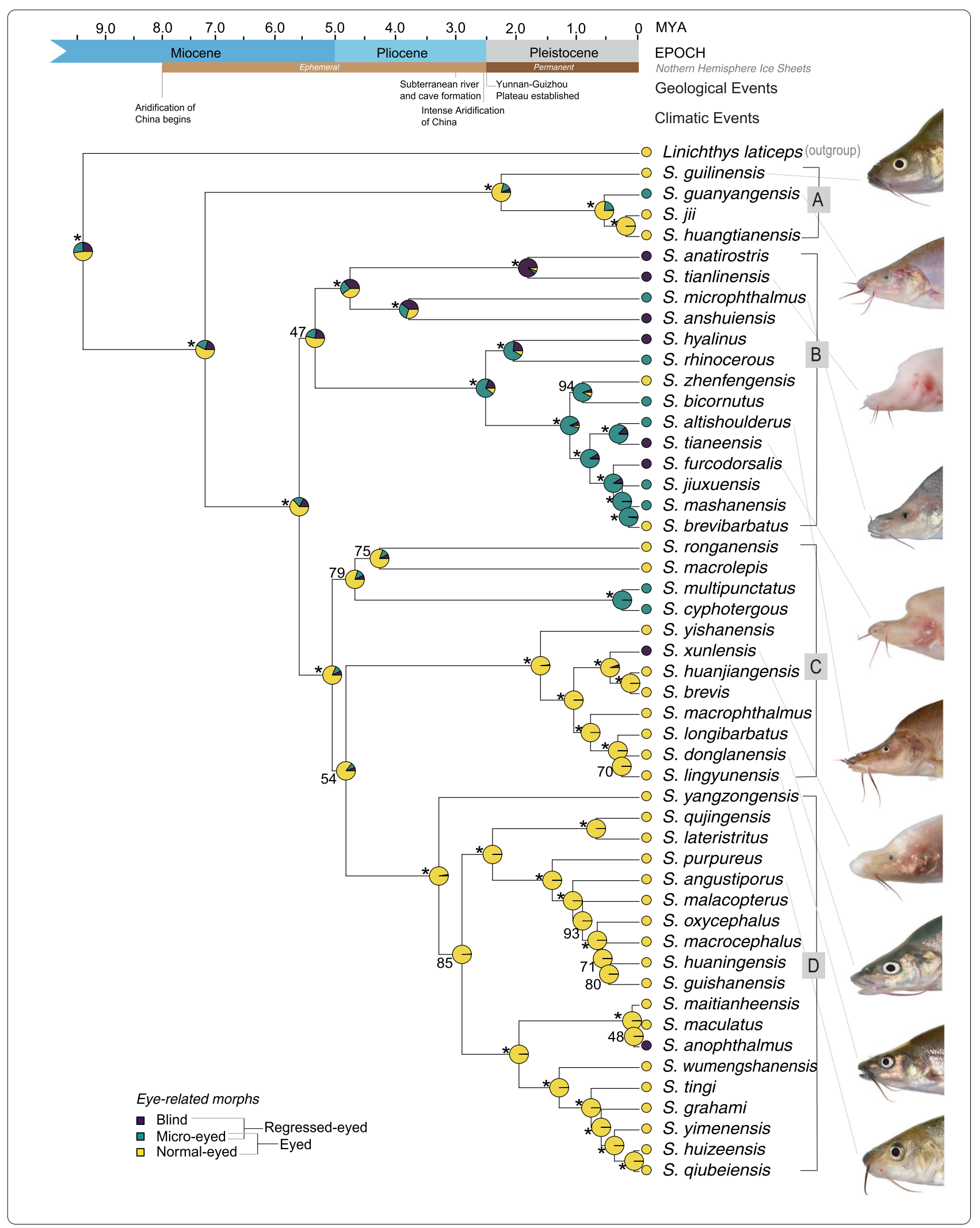




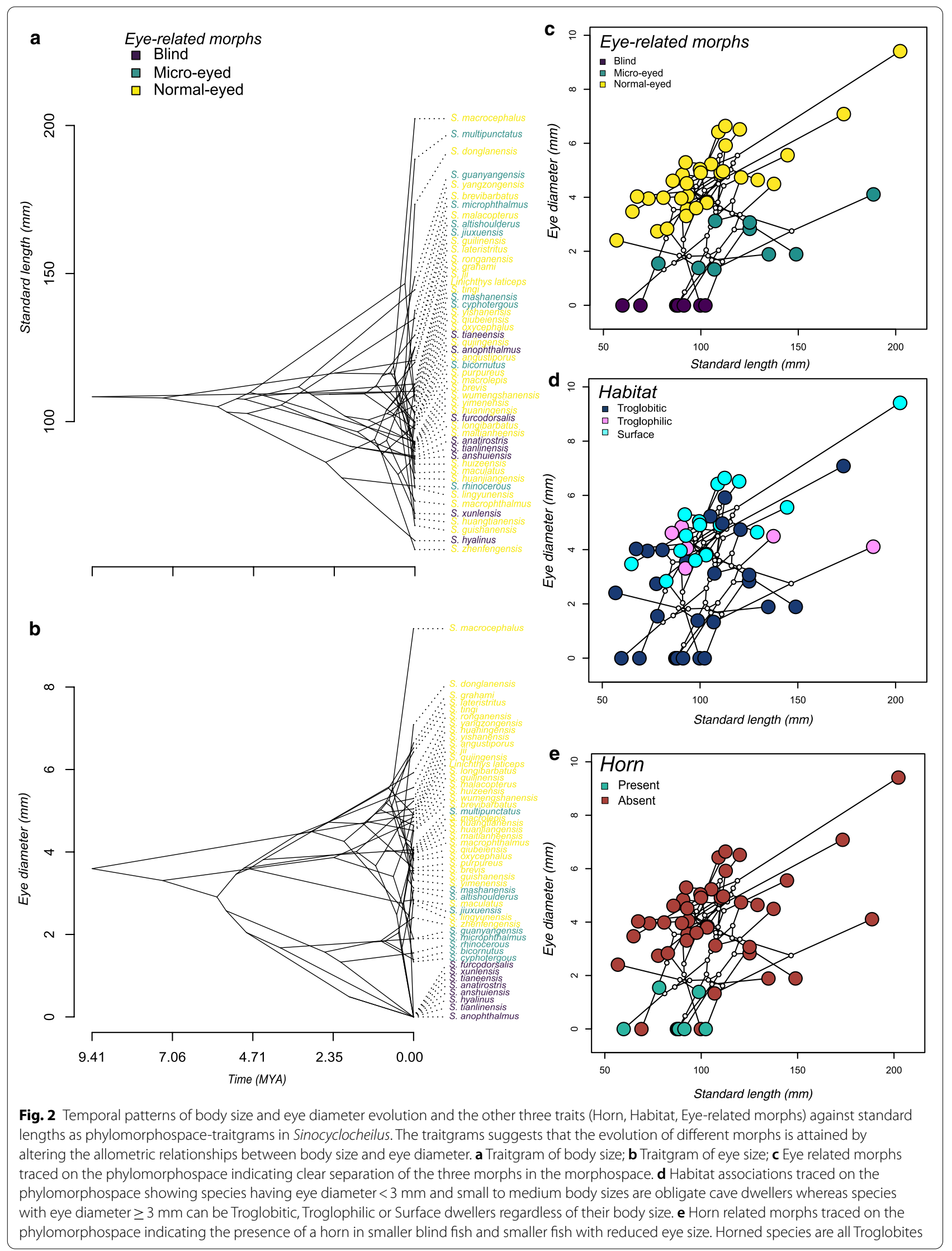


(See figure on next page.)

Fig. 3 a A specimen of Sinocyclocheilus altishoulderus indicating the position of 15 landmarks (red: larger points indicated by numbers 1-15) and 180 semi-landmarks (Orange: smaller point) used for the calculation of Procrustes coordinates and traditional linear measurements (SL standard length, ED eye diameter and sED standardized eye diameter) used in the geometric morphometric analyses. $\mathbf{b}$ PCA showing the variation in body shape of the genus Sinocyclocheilus traced with eye morphology, habitat occupation, existence of horn. PC1 and PC2 accounts for $32 \%$ and $17 \%$ of the variance respectively. A shift from the fusiform shape of the Normal-eyed surface forms to a more "boxy" form of the Micro-eyed and Blind forms is evident

for approximately $32 \%$ of the variance in the dataset (see Additional file 1: Table S1), tended to distinguish the slender Normal-eyed species on the left and Micro/ Blind species on the right, which were characterized by changes in shape and widening of the anterior dorsal area between mouth and beginning of the dorsal fin of their body, resulting in a shift from the fusiform shape of the Normal-eyed forms to a more "boxy" form of the Micro-eyed and Blind forms. The second PC, which explained approximately $17 \%$ of the variance in the dataset, emphasized the differences in the type of dorsoventral broadening of the mid-section between morphs, with a shortening of the tail region (Fig. 3b). The variation in this axis is very high among the Micro-eyed and Blind forms when compared to the Normal morphs. Multivariate Analysis of Variance (MANOVA) showed that Eye-morphology and Horn bearers were significantly different in body shape; Body shape was not significantly associated with habitat type. (Eye-morphology, $\mathrm{F}=15.28$, $\mathrm{p}=0.001$; Horn, $\mathrm{F}=6.14, \mathrm{p}=0.016$; Habitat, $\mathrm{F}=1.72$, $\mathrm{p}=0.153)$.

\section{Rates of morphological evolution}

The multiple-rate model of evolution provided the best fit to the data for all three quantitative traits $(\triangle \mathrm{AIC}=3.6$, 14.2 and 8.3 respectively for ED, sED and SL; Table 1 ), indicating that the evolution of different Sinocyclocheilus morphs was associated with significant changes in their evolutionary rates. However, there were intriguing differences between traits in their rates (Table 2). The rates of evolution of eye diameter and standardized eye diameter were similar between Normal-eyed and Micro-eyed species, but increased between 5.5 and 20.8 times during shifts towards Blind species.

\section{Spatial patterns in Sinocyclocheilus evolution}

Geophylogeny represents the phylogeny overlaid across the geographic location of each species, where phylogenetic clustering is evident across the landscape. Considering the distribution of Sinocyclocheilus, we see mainly a pattern where the early-diverging, Normal-eyed morphs are placed in the east, a substantial portion of Blind/ Micro-eyed (Regressed-eyed) species are in the center, and Normal-eyed morphs are predominant towards the western mountains (Fig. 4a and b). Although few in numbers, horned species (all horned species are contained in Clade B) seems to be scattered within their distribution range (Fig. 4c).

\section{Discussion}

\section{Habitat utilization in context of eye-morphology}

Integrating evolution of eye size and habitat manifests interesting and previously unrecognized evolutionary patterns in the evolution of Sinocyclocheilus. The eyedsize based ancestral reconstruction suggests the base of the phylogeny is most likely an eyed species (i.e. Normalor Micro-), but habitat reconstructions places, with high probability, Troglobitic species at the base (Additional file 1: Fig. S4). This suggests an ancestral eyed-species evolved a Troglobitic habit before becoming blind. This may be an example of preadaptation in Sinocyclocheilus, i.e., the advancement of a functional change with little or no evolutionary modification [43]. In Astyanax cavefish, surface-dwelling forms are scotophilic; they prefer to remain away from direct light, suggesting that scotophilia may be preadaptive for colonizing the dark cave environment [44], but there are no confirmed cases of character reversal in Astyanax. In Sinocyclocheilus, since an ancestral (eyed) species demonstrated preference for the cave habitat, this preadaptation to darkness may hint towards why certain species tend to become cave-dwellers while others do not. This pattern is supported by two principal lines of evidence. First, most of the early-diverging species are eyed, and Troglobitic (except for one species with an unusual eye-related polymorphic condition that we discuss below). Second, the most westward group (Clade D; Normal-eyed Surface fish), re-colonized caves whenever cave habitats were available within that area, suggesting a strong predisposition for cave-dwelling across all Sinocyclocheilus. In other words, when caves were available, members of Sinocylocheilus, irrespective of eyerelated condition, prefer cave dwelling.

In the context of Sinocyclocheilus, the preference for caves may be not only a preference for darkness but also a preference for depth, in search of retreating water for survival. In karstic environments, where surface desiccation is common, a preference for such deeper refuges may have conferred a fitness dividend. In the presence of an array of subterranean waterways, such a predisposition would have given rise to the eye-regressed forms 


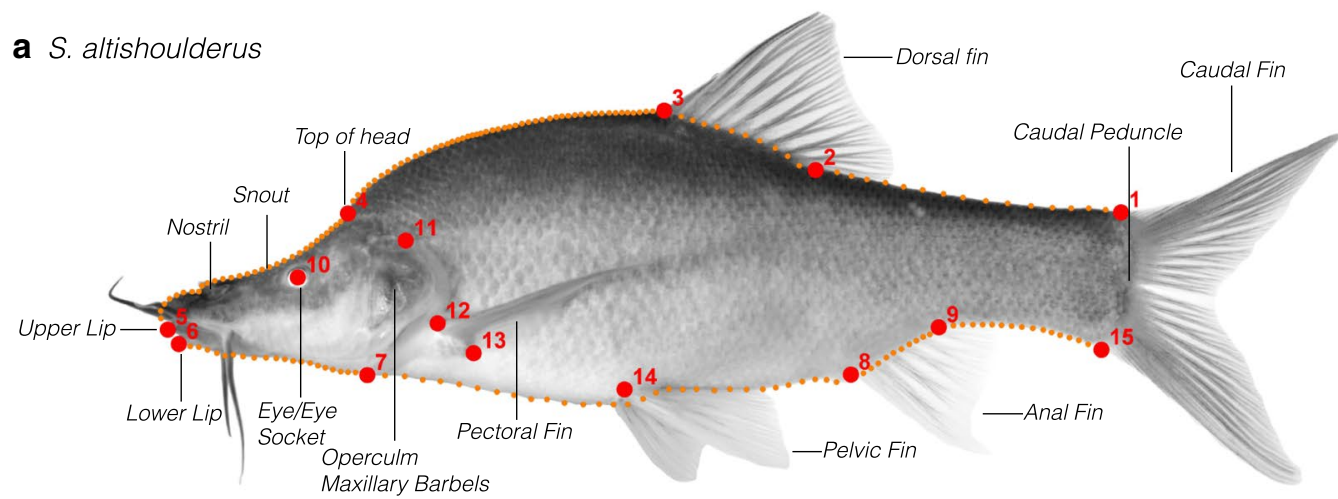

b
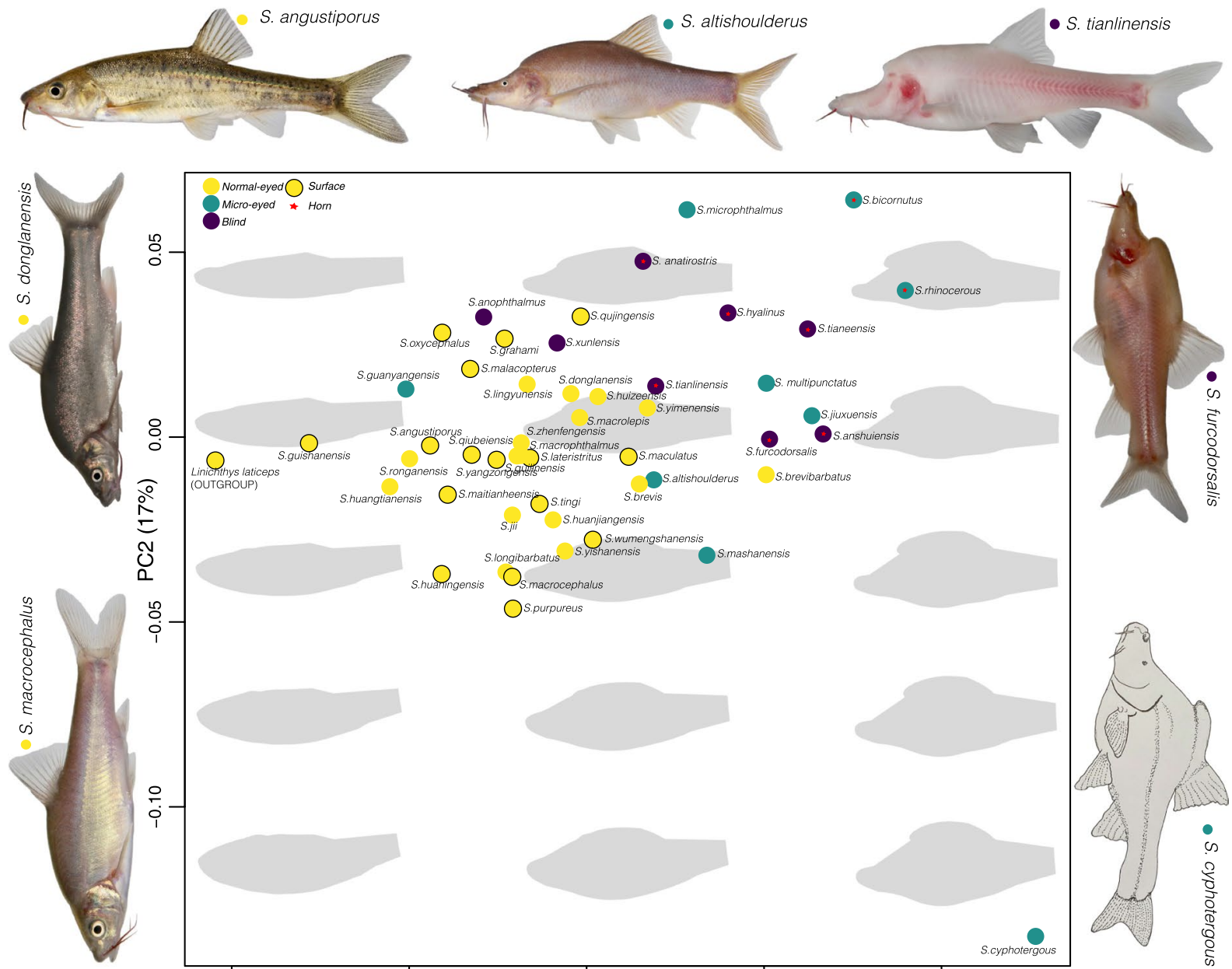

웅
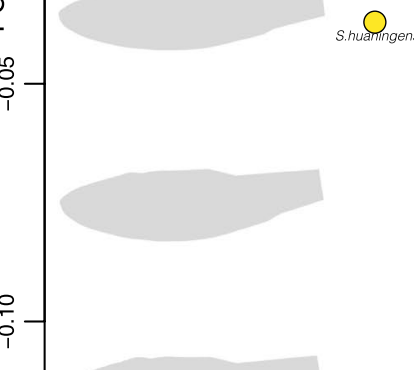

S.longibarbatus s.yshanensis

$$
\text { S.p.purpureus }
$$
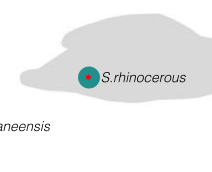
Table 1 Model fit and estimated Brownian rate parameters for three traits (ED, SED and $S L$ ) in eye related morphs of Sinocyclocheilus

\begin{tabular}{|c|c|c|c|c|c|}
\hline \multirow[t]{2}{*}{ Trait } & \multicolumn{2}{|c|}{ Single rate model } & \multicolumn{2}{|c|}{ Multiple rate model } & \multirow[t]{2}{*}{$\triangle \mathrm{AIC}$} \\
\hline & $\mathrm{AlCC}$ & AlC weights & $\mathrm{AlCC}$ & AIC weights & \\
\hline 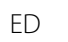 & 254.9848 & 0.142077010 & 251.3885 & 0.857923 & 3.596290 \\
\hline$=D$ & 249.0874 & 0.000806064 & 234.8423 & 0.999194 & 14.245083 \\
\hline$S L$ & 532.7615 & 0.015145208 & 524.4119 & 0.984855 & 8.349620 \\
\hline
\end{tabular}

Multiple-rate models of evolution providing the best fit to the data for all three quantitative traits with $\triangle \mathrm{AIC}>2$

Table 2 Model averaged rate parameters for the measured traits in eye related morphs of Sinocyclocheilus

\begin{tabular}{llrc}
\hline Trait & \multicolumn{2}{l}{ Model averaged rate } \\
\cline { 2 - 4 } & Blind & Micro-eyed & Normal-eyed \\
\hline SL & 44.0468 & 1775.2360 & 1465.4660 \\
ED & 23.2990 & 3.9025 & 4.5091 \\
SED & 44.6336 & 1.4413 & 2.8972 \\
\hline
\end{tabular}

Normal-eyed and Micro-eyed species indicate similar evolutionary rates with marked shifts towards Blind species

living close to, or associated with, caves that are characteristic of the genus.

Furthermore, apart from the Troglobitic and Troglophilic species of Clade D, some of the putative Surface species of this clade are often observed at the entrances of caves or at windows to subterranean rivers [20]. Future ecological studies may reveal that some taxa recognized as Surface forms may indeed be Troglophilic, bolstering the notion that Sinocyclocheilus are predisposed to seek refuges in deeper waters of karstic caves.

Resource utilization plays a key survival role in harsh environments [45], as maybe the case for Sinocyclocheilus. Some Troglophilic eyed-species like S. altishoulderus, S. donglanensis [21], S. microphthalumus, S. malacopterus [46] and S. longibarbatus (personal observation), are known to emerge from caves during the high-water season, presumably to feed and breed. This explains dependence on the cave as a diurnal refugium, from where these species can exploit the surface habitats at night. Strategies such as this, where multiple resources are utilized concurrently, points to the adaptability of some Sinocyclocheilus species, resulting in their persistence in a harsh and changing environment. In this sense, the cave entrances are possibly an ecotone that is important during Sinocyclochelius diversification.

\section{Adaptations in the light of geophylogeny}

Early-diverging Sinocyclocheilus (Clade A) are NormalEyed, predominantly cave dwelling and non-horned species from the eastern region of their distribution. The ancestral morphs (at the base) suggest that the affinity to caves would have evolved early and is present in most Sinocyclocheilus. The clade comprising of early-diverging species is from the east of the Sinocyclocheilus distribution, i.e. the He Jiang and Gui Jiang basin in northeastern Guangxi, and hence, it seems that the diversification of these fishes occurred from east to west (Fig. 4a).

Within this predominantly Normal-eyed clade (Clade A), there is an exception: Sinocyclocheilus guanyangensis, a species that we coded as Micro-eyed, has Normal-Eyed, Micro-eyed and effectively Blind morphs within the same population, thus it is polymorphic for this trait. But these blind morphs have their eyes completely covered by skin and the eye is not itself degenerate. This kind of condition has been observed also in several other congeners (S. xunlensis and S. flexuosdorsalis-latter species not included in our analysis due to lack of molecular data), but is uncommon. This suggests a degree of polymorphism for this trait, indicating that the earliest ancestors of Sinocyclocheilus may have been able to lose or gain eyes relatively easily as an adaptation to local conditions. This pattern appears several times within this cave-driven diversification.

The major adaptation for cave dwelling evolves predominantly in the expansive karstic area in northwestern Guangxi (associated with the Liu Jiang basin and Hongshui river basin joining the main Xijiang River system from the North), in Clade B, the southeastern corner of Guizhou province (upper reaches of Hongshui River) and the northeastern plateau of Yunnan province. This region can be considered the center for novel adaptations for Sinocyclocheilus, where these fishes express their full morphological diversity, blindness, micro-eyedness, and their remarkable horns. In the shape-related analyses, these species cluster on the right of morphospace (Fig. 3b). The deeper caves and extensive subterranean river system associated with the Guangxi plains [20] would have facilitated this extensive adaptive diversification (Fig. 4).

The karstic northwestern region to which the Guangxidominated clade (Clade B) belong to experiences drought conditions during much of the year, and one of the major sources of rain for the region is from storms sweeping from the southeast, which are strong enough to persist through the vast plains of Guangxi, mainly from April to August [20]. During the intervening dry periods, however, these fishes seem to have found refuge in the subterranean caves. The morphologically most diverse clade inhabiting the region where the climatic conditions are most unfavorable to surface fish reinforces the notion that Sinocyclocheilus adapted to life in caves as climatic refuges [20]. 

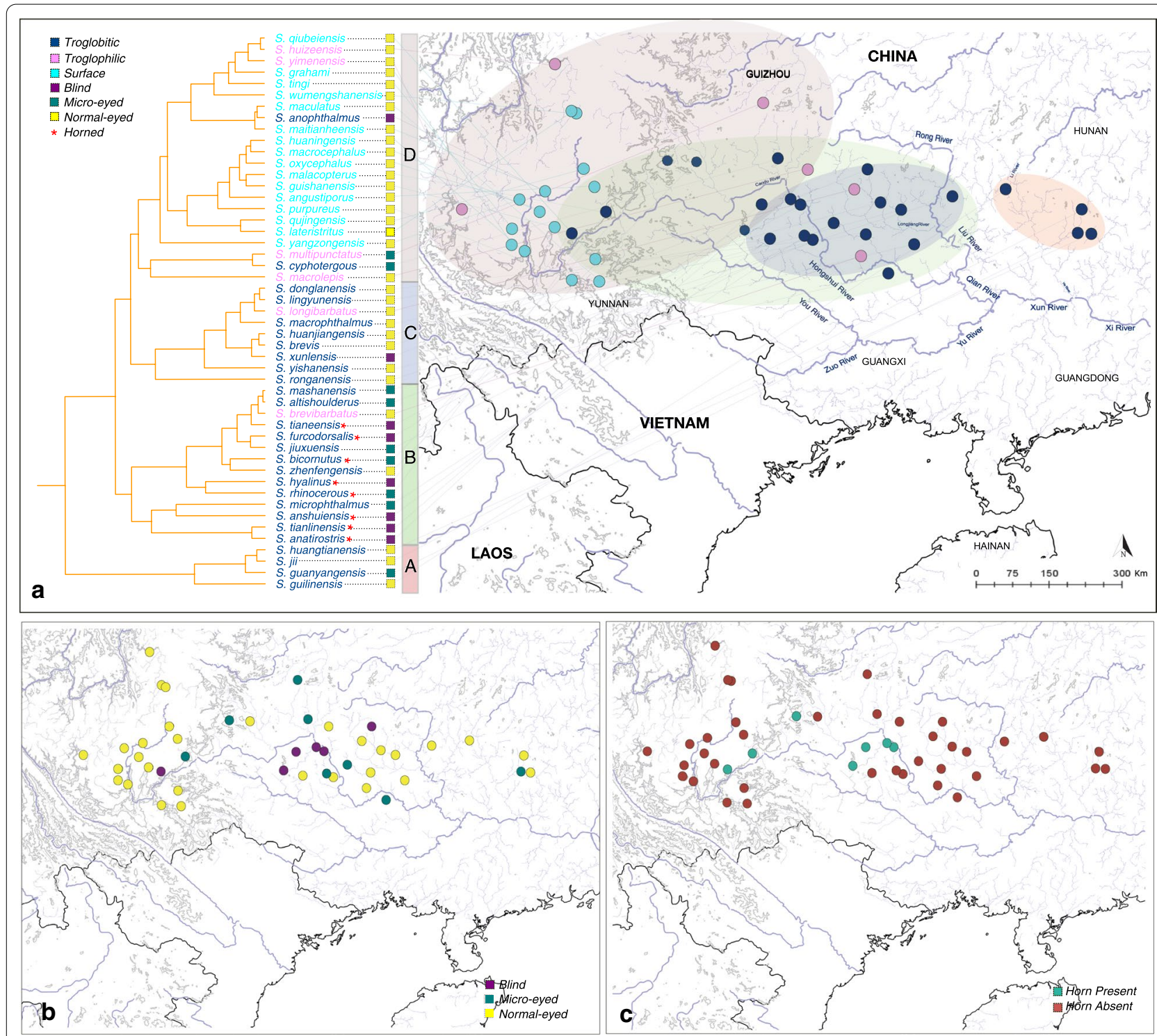

Fig. 4 Geophylogeny, the phylogeny laid across the geographic distribution of the species considered in the analysis, with habitats (a), eye-related morphs (b) and horn-existence (c). A pattern where early-diverging, Normal-eyed, Troglobitic species are placed in the east, predominantly Blind/ Micro-eyed/Normal-eyed, Troglobitic species in the center and Normal-eyed, Surface dwelling species towards the western mountains is evident, indicating an east to west dispersion of the genus Sinocyclocheilus across South and South western China. Eye specializations mostly occurred in Clade B, and horn evolution occurred exclusively in Clade B, within the Central range of the Sinocyclocheilus distribution. [Vector files were obtained from a public domain map data at http://naturalearthdata.com]

The distribution of Clade $\mathrm{C}$, characterized by mostly Normal-eyed but Troglobitic species largely overlaps Clade B. In Clade C, the single Blind species (S. xunlensis) and the two Micro-eyed species (S. cyphotergous and S. multipunctatus) are shown within a narrow geographic area on the Liu Jiang and Hongshui river system (Fig. 4b).

The clade containing species to the west (Clade D), predominantly on the hilly terrain of Yunnan plateau, are Normal-eyed Surface species lacking horns (Figs. 1 and 4). However, when cave habitats and subterranean river systems are available, some of these surface adapted species have become Troglobytic or Troglophilic. The Troglobytic species occurring in the region, S. anophthalmus, is blind. However, this Blind species stands clustered with the Normal-eyed morphs in morphospace, signifying that the shape of the species has not extensively changed, possibly due to recent (Pleistocene) invasion of the cave habitat by a Normal-eyed ancestor 
(Fig. 1) suggesting that the time since entering the cave has been insufficient to change into the characteristic box-like shape of the Blind species of Clade B.

Horn distributions show several peculiar trends. In most Sinocyclocheilus species, a prominent hump is present [47]. However, this hump is markedly low in the Normal-eyed surface-inhabiting species of the Yunnan clade (Fig. 4). For species that bear a horn, this structure occurs in the region in which the dorso-frontal hump is present, and always occurs anterior to the hump, at the dorsal occipital margin. The exception to this is $S$. cyphotergous (Clade C), where a horn-like structure occurs close to the anterior end of the dorsal fin, on top of the hump. This species (in Clade C) is phylogenetically separate from other horned species, suggesting that the origins of the "horn" for this species is evolutionarily different from the other horned species (Additional file 1: Fig. S2). Though the function of the horn remains unknown, functions such as protection of head, a role in sexual selection, and anchoring in strong current have been suggested [20,48]. In S. cyphotergous, this structure may be similar to those in other species in function.

\section{Troglomorphism and rates of evolution}

The rates of evolution of various traits show some incongruent (non-allometric, $R=0.034, p=0.82$ ), but interesting patterns that can be explained in the context to adaptations to a Troglobitic condition. The rates of evolution in eye diameter are similar between Normal- and Micro-eyed species but, increase dramatically (5.5-20.8 times) with shifts towards blind forms. However, body size evolution for these morpho-groups shows a reversed pattern, with a 0.03 decrease in body size evolution in the Blind morphs compared to the eyed-morphs. These patterns in rate variation suggest that the evolution of Blind morphs to a Troglobitic habitat were simultaneously associated with an increase in the rate of evolution of eye-size degeneration and a decrease in the rate of body size evolution. The smaller body size resulting from a sluggish rate of change facilitates both navigation within constricted spaces and sustenance on a limited supply of resources as expected in subterranean habitats [49].

Much of our collective knowledge of the patterns and mechanisms of regressive evolution come from studies of animals that have colonized the subterranean biome. Within this group, several studies have focused on the Astyanax mexicanus [5, 50-53]. This natural animal model system comprises multiple cave-adapted morphs and a surface-dwelling morph that resides in or near the caves themselves [54]. Since the discovery of this cavefish in 1936, studies have provided insight to the developmental and genetic bases for cave-associated traits [55-62]. Indeed, much of this insight has emerged from the interbreeding studies of conspecific cave and surface morphs (reviewed in [63]). However, several aspects of regressive evolution and troglomorphic adaptation remain unresolved. Owing to several of the differences with Astyanax, we argue that Sinocyclocheilus, in the form of a multi-species evolutionary model system, is well-positioned to provide important new insights to broader patterns of diversification and adaptation in cave-dwelling organisms.

In conclusion, Sinocyclocheilus fishes seem to have occupied the cave habitats in response to drying associated with aridification of China during late Miocene and the Pliocene. The early-diverging clades of these fishes are located in the eastern corner of their distribution, predominantly in Guangxi region. The most prominent cave-adaptations such as reduction and loss of eyes and evolution of horns also seem to have taken place in clades that are predominantly found in Guangxi and Guizhou provinces and are associated with the extensive subterranean cave system in the area. There is eye-related and Horn related two-dimensional shape variation seen within the diversification, which is statistically significant. The habitat related shape variation is not significant, this is because Normal-eyed species are in most clades are also troglobitic, except in Clade D, the westward clade in this diversification. The lineages leading to Blind morphs are associated with significant rate shifts such as slow down of body size evolution and significant increase of eye-regression; this is possibly associated with the scarcity of resources. The integration of morphology, phylogeny, rate analyses, dating and distribution show not only several remarkable patterns of evolution, but also interesting exceptions to these patterns, which signify the diversification of Sinocyclocheilus as a unique multi-species model system to study evolutionary novelty.

\section{Methods}

We carry out an analysis to explore the key patterns of morphological evolution, habitat utilization and to determine how these patterns are dispersed across the landscape in Sinocyclocheilus cavefishes. We first infer a phylogeny based on mitochondrial DNA (mtDNA) which we use to determine patterns of evolution. We also determine shape associations of these species in terms of eye morphology, habitat and horn existence using a landmark based analysis. We calculate the rates of evolution of body size in relation to eye size evolution, habitat utilization and horn evolution. We use ancestral reconstructions on a timing-tree to determine the evolutionary patterns of eye regression, habitat occupation and horn evolution. Finally we trace the how these patterns are dispersed across the landscape using a geophylogeny. This framework will provide a comprehensive understanding 
of the major patterns of morphological evolution for Sinocyclocheilus cavefishes.

\section{Phylogeny estimation}

We compiled sequence data from GenBank for two mtDNA fragments (NADH4 and cytb) of 39 Sinocyclocheilus species, and five outgroup species Linichthys laticeps, Gymnocypris eckloni, Gymnocypris przewalskii, Labeo batesii, Puntius ticto. In addition, we generated sequence data for the NADH4 and cytb gene fragments of nine additional Sinocyclocheilus species (Additional file 1: Table S2). For these species, total genomic DNA was extracted using the DNeasy Blood and Tissue Kit (Qiagen Inc., Valencia, CA) following the manufacturer's protocols. DNA was amplified in $25-\mu \mathrm{L}$ volume reactions: $3 \mathrm{mM} \mathrm{MgCl} 2,0.4 \mathrm{mM}$ of dNTP, $1 \mathrm{X}$ buffer, $0.06 \mathrm{U}$ of Taq DNA Polymerase, $2 \mathrm{mM}$ of each primer. Thermocycling conditions included an initial step at $94{ }^{\circ} \mathrm{C}$ for $3 \mathrm{~min}$, followed by 35 cycles at $45 \mathrm{~s}$ at $94{ }^{\circ} \mathrm{C}, 1 \mathrm{~min}$ at $46-50{ }^{\circ} \mathrm{C}$ and $45 \mathrm{~s}$ at $48-56{ }^{\circ} \mathrm{C}$, and a final step at $72{ }^{\circ} \mathrm{C}$ for $5 \mathrm{~min}$. PCR products were electrophoresed in a $1.5 \%$ agarose gel, stained with ethidium bromide and visualized under UV light. Successfully amplified products were purified using MicroconTM Centrifugal Filter Units (Millipore, Billerica, MA, U.S.A.). Sequencing reactions were carried out in $10 \mu \mathrm{l}$ solutions including the following final concentrations: $5 \mathrm{ng} / \mu \mathrm{l}$ of template DNA, $0.5 \mu \mathrm{l}$ of Big DyeTM (Applied Biosystems Inc., Foster City, CA, U.S.A.), $0.2 \mu \mathrm{M}$ of each primer and $0.1 \mathrm{X}$ of reaction buffer. The final product was purified using SephadexTM G-50 (GE Healthcare Bio-Sciences AB, Uppsala, Sweden) for sequencing. Forward and reverse strands were reconciled using Staden v.1.6.0 [64].

Sequences from both genes were concatenated and aligned unambiguously using ClustalW [65], as implemented in MEGA v. 6.0 [66], for a total alignment length of 2167 bp. We used jModelTest v.2.1.10 [67] to determine the best models of evolution for each fragment, which were implemented in BEAST v.1.10.4 [68] as a partitioned analysis to estimate the phylogenetic relationships and relative divergence times within Sinocyclocheilus. Due to the lack of a fossil record for the Sinocyclocheilus, we used two alternative approaches to estimate divergence times in Sinocyclocheilus. First, we used a "standard clock" estimate for fish mitochondrial DNA of $2 \%$ per million years [19], as the main approach of this study. We also used two calibration points $(\mathrm{C} 1$, C2) based on the approach of $\mathrm{Li}$ et al. [12] for exploring potential differences in estimates of divergence times using different approaches. We used a strict molecular clock and a calibrated Yule tree prior, as well as a $\mathrm{GTR}+\mathrm{I}+\mathrm{G}$ for each partition and ran the analysis for 20 million generations using the Cipres Science Gateway
Server [69]. Convergence was assessed by inspecting the log-output file in TRACER v.1.7.1 [70] and by ensuring ESS values were above 200 . The first $10 \%$ of the trees were discarded, and the post burn-in trees were used to infer the maximum clade credibility tree using TreeAnnotator v.1.10.4 [71]. The maximum clade credibility tree, as well as a set of 1000 post burn-in topologies, were retained for further analyses (see below).

\section{Morphometric data acquisition and analyses}

To determine shape associations of these species, we assembled a database of images of referenced specimens of adult Sinocyclocheilus from scaled photographs (we only used images that contained scale-bars), which were complemented by species that we photographed (Additional file 1: Table S3), and we measured the left side (lateral view) of the fish. The final dataset included 90 images (54 photographs by us and 36 from photographs from previous studies) for 50 species, which included $65 \%$ of the total number of described Sinocyclocheilus species. These images were used for geometric morphometrics analyses, which were based on 15 landmarks (Fig. 3a) and 180 sliding semi-landmarks, obtained using tpsDig v. 2.16 [72]. Semi-landmarks were collected as curves outlining the body. These data were subsequently reduced to equidistant landmarks, and defined as semi-landmarks using tpsUtil v. 1.46. These images were treated with the Unbend function of tpsUtil to remove bending effects [73]. We then slid the landmarks using the bending energy method [74] implemented in GEOMORPH v.3.2.0 [75]. The landmark coordinates were aligned using a generalized Procrustes superimposition analysis [76], and a principal component analysis (PCA) was used to evaluate shape variation within the sample. Multivariate analysis of variance (MANOVA) was performed to determine if there were statistically significant differences in body shape among the below mentioned categorizations tested (Eye-morphology, Habitat and Horn).

Multiple images for the same species were used to obtain the landmarks (1-3 photographs per each species, Additional file 1: Table S3) and the mean of their Procrustes coordinates were calculated to be used in later analyses. We also obtained traditional linear measurements, namely standard length (SL), eye diameter (ED) and standardized eye diameter ( $\mathrm{SED}$, calculated as the ratio between ED and $\mathrm{SL}$ ). We used zero for the eye diameter for the blind fishes.

\section{Morphological and habitat evolution}

Since shape variation in Sinocyclocheilus cavefishes occurs mostly in the anterior end of the fish and as one of the major features leading to this is eye-related, we considered the absence or the size of the eye (when present) 
as a proxy for the categorization of morphs. Since the eye size has an allometric association with body size, we used the standardized eye diameter (sED) in placing them into three morphological categories: Blind (eye absent); Micro-eyed ( $\leq 3.0 \mathrm{~mm}$ ); Normal-eyed $(>3.0 \mathrm{~mm}$ ) respectively. For ease of discussion, we considered Blind and Micro-eyed together as Regressed-eyed; Micro-eyed and Normal-eyed together as eyed species.

Sinocyclocheilus were also categorized based on their habitat as Troglobitic, Troglophilic, and Surface species. Troglobitic species live in an obligatory association with caves and are not sampled outside of caves. Caves, as meant here represent roofed-caves, submerged caves, and subterranean waterways that form windows intermittently with the surface. Troglophilic species live in a close association with caves and are sampled both in the vicinity of cave entrances and within caves. Finally, Surface species are found in habitats even when a cave is not found in close proximity and live in normal streams ponds and lakes as typical fish do, but they could venture into caves (underground water bodies) during unfavorable periods, when water is only available in caves. It should be noted here that this categorization is strictly habitat based and not morphology based (for instance, there are Normal-eyed species that are Troglobitic, Troglophilic or Surface). These habitat associations are based on published literature $[20,21]$ and personal observations as outlined in Additional file 1: Table S2.

To infer the number and timing of evolutionary shifts within eye-related morphs, horn distribution and the habitat type, we used stochastic character mapping [77, 78], as implemented in the make.simmap (model="ER") function in PHYTOOLS. We tested multiple models for the ancestral state reconstruction, the ER model gave the highest likelihood. On each of the 1000 post burnin trees obtained from BEAST, we used stochastic character mapping to generate 100 potential histories. This approach therefore considers uncertainty both in the evolutionary history of the traits as well as in the inferred topology of the phylogeny.

The landmark coordinates obtained were aligned using a generalized Procrustes superimposition analysis [76], and a principal component analysis (PCA) was used to explore shape variation within the sample. In addition, we described the eye-related morphological variation in Sinocyclocheilus by estimating ancestral states of SL and ED and visualizing them using traitgrams [79] as implemented in the phenogram function in PHYTOOLS [80] using the maximum clade credibility tree. We also visualized the evolution of both traits simultaneously using a phylomorphospace [81] using the phylomorphospace function in PHYTOOLS. Furthermore, to assess the allometric relationship of the size of the eye against the standard body length we also performed an allometric regression between SL and SED.

\section{Evolutionary rate variation in eye related morphs}

We tested whether the evolutionary rates of the studied continuous traits (SL, ED, sED) are significantly different in different morphs. We used 100 potential trait histories from stochastic character mapping and then fit two alternative models of evolution on each studied trait, one that fixes the rate of evolution to be identical between morphs against an alternative model in which the morphs have separate rates. We calculated the Akaike Information Criterion for small sample size (AICc) from the maximum likelihood estimate on each tree using the brownie.lite function in PHYTOOLS. Finally, to incorporate uncertainty about model choice into the parameter estimate, we calculated model-averaged estimates of evolutionary rates for each morph using the Akaike weights from the mean AICc scores. Unless otherwise indicated, all analyses were conducted using R v. 3.6.2 [82].

\section{Geophylogeny analyses}

To determine the patterns of evolution of eye-regression, habitat utilization and horn existence in the context of Sinocyclocheilus distribution we built a geophylogeny on which we traced these traits. We, however, could not carry out a formal biogeographical analysis, given that their high endemism and the complex pattern of underground connections between caves limits the establishment of reasonable biogeographical areas. However, we assessed the geographical structuring of Sinocyclocheilus diversification by building the geophylogeny on GenGIS v. 2.5.3 [83] based on the maximum credibility tree.

\section{Abbreviations}

mtDNA: Mitochondrial DNA; SL: Standard length; ED: Eye diameter; sED: Standardized eye diameter; PCA: Principal component analysis; MANOVA: Multivariate analysis of variance; AICc: Akaike information criterion; GXU: Guangxi University.

\section{Supplementary Information}

The online version contains supplementary material available at https://doi. org/10.1186/s12862-021-01776-y.

Additional file 1: Figure S1. Ancestral character state reconstruction using stochastic character mapping for the eye-trait morphology (Blind, Micro and Normal-eyed morphs) on a time calibrated phylogeny. Figure S2. Ancestral character state reconstruction using stochastic character mapping for the horn related trait (presence/absence of horn) on the phylogeny of the genus Sinocyclocheilus. Figure S3. Temporal patterns of eye diameter evolution and the other three standardized traits (Horn, Habitat, Eye-related morphs) against standard lengths as phylomorphospacetraitgrams in Sinocyclocheilus. Figure S4. Ancestral character state reconstruction using stochastic character mapping for habitat occupation (Troglobitic, Troglophilic and Surface) on the phylogeny of the genus Sinocyclocheilus. Table S1. Calculated Principal Component values (PC1, 
PC2 and PC3) of all the specimens used in the current analysis. Table S2. Species information and GenBank accession numbers of two mtDNA fragments (NADH4 and cytb) of 49 Sinocyclocheilus species. Table S3. Information of digitized images used in the morphometric geometric analysis

\section{Acknowledgements}

We thank the following individuals: Rohan Pethiyagoda for helpful comments on an earlier version of the manuscript; Shipeng Zhou, Bing Chen, Dan Sun, Jayampathi Herath and Amrapali Rajput for assistance in the field.

\section{Authors' contributions}

MM, MRP, TRM, YWL, JG, JY conceptualized the research and designed the methodology. YWL, CHF, MTR, MM, GE, JY conducted fieldwork and curated the data. TRM, YWL, MRP, GE, GS, MM carried out formal analysis. MM, MRP, JG, TRM, YWL, GE, GS, wrote the original draft. MM and MRP supervised MTR, YWL. $M M$ and JY acquired funding. MM, MRP, TMR, GE and YWL made figures. MM carried out fish photography and drawing. All authors reviewed and edited the draft. All authors read and approved the manuscript.

\section{Funding}

Funding for this study is provided by (1) Guangxi University Startup Funding to MM for fieldwork, lab work, analyses and supporting TRM, YWL, CHF (2) National Natural Science Foundation of China (\#31860600) to JY for fieldwork (3) Guangxi Natural Science Foundation (\#2017GXNSFFA198010) to JY for research work. These funding bodies played no role in the design of the study and collection, analysis, and interpretation of data and in writing the manuscript.

\section{Availability of data and materials}

All data generated or analyzed during this study or the sources of data (Genbank) are included in this published article.

\section{Ethics approval and consent to participate}

Methods of sampling approval by the Ethics Committee of Guangxi University. Field sampling approval through the Guangxi Provincial Government.

\section{Consent for publication}

Not applicable.

\section{Competing interests}

The authors declare that they have no competing interests.

\section{Author details}

${ }^{1}$ Guangxi Key Laboratory for Forest Ecology and Conservation, College of Forestry, Guangxi University, Nanning, Guangxi, People's Republic of China. ${ }^{2}$ Key Laboratory of Environment Change and Resource Use, Beibu Gulf, Nanning Normal University, Nanning, Guangxi, People's Republic of China. ${ }^{3}$ Faculty of Science, University of Peradeniya, Peradeniya, Sri Lanka. ${ }^{4}$ Department of Organismal Biology \& Anatomy, University of Chicago, Chicago, IL, USA. ${ }^{5}$ Department of Biological Sciences, University of Cincinnati, Cincinnati, $\mathrm{OH}$, USA. ${ }^{6}$ Departamento de Zoologia, Universidade Federal do Paraná, Curitiba, PR, Brazil.

\section{Received: 30 October 2020 Accepted: 8 March 2021}

Published online: 17 March 2021

\section{References}

1. Ginet R, Decou V. Initiation à la biologie et à l'écologie souterraines. Paris: J.-P. Delarge; 1977.

2. Camacho AI. The natural history of biospeleology. Editorial CSIC-CSIC Press; 1992.

3. Soares D, Niemiller ML. Sensory adaptations of fishes to subterranean environments. Bioscience. 2013;63:274-83.

4. Yoshizawa M. Behaviors of cavefish offer insight into developmental evolution. Mol Reprod Dev. 2015;82:268-80.
5. Dowling TE, Martasian DP, Jeffery WR. Evidence for multiple genetic forms with similar eyeless phenotypes in the blind cavefish, Astyanax mexicanus. Mol Biol Evol. 2002;19:446-55.

6. Jingcheng $R$, Weicheng $Y$. A review of progress in Chinese troglofauna research. J Resour Ecol. 2015;6:237-46.

7. Strecker U, Faúndez VH, Wilkens H. Phylogeography of surface and cave Astyanax (Teleostei) from Central and North America based on cytochrome b sequence data. Mol Phylogenet Evol. 2004;33:469-81.

8. Zhao Y, Zhang C. Cavefishes: concept, diversity and research progress. Biodivers Sci. 2006;14:451

9. Gross JB, Protas M, Conrad M, Scheid PE, Vidal O, Jeffery WR, et al. Synteny and candidate gene prediction using an anchored linkage map of Astyanax mexicanus. Proc Natl Acad Sci USA. 2008;105:20106-11.

10. Culver DC, Kane TC, Fong DW. Adaptation and natural selection in caves: the evolution of Gammarus minus. Cambridge: Harvard University Press; 1995.

11. Jeffery WR. Cavefish as a model system in evolutionary developmental biology. Dev Biol. 2001;231:1-12.

12. Li Z, Guo B, Li J, He S, Chen Y. Bayesian mixed models and divergence time estimation of Chinese cavefishes (Cyprinidae: Sinocyclocheilus). Chin Sci Bull. 2008;53:2342-52.

13. Yang J, Chen X, Bai J, Fang D, Qiu Y, Jiang W, et al. The Sinocyclocheilus cavefish genome provides insights into cave adaptation. BMC Biol. 2016:14:1-13.

14. Hart PB, Niemiller ML, Burress ED, Armbruster JW, Ludt WB, Chakrabarty P. Cave-adapted evolution in the North American amblyopsid fishes inferred using phylogenomics and geometric morphometrics. Evolution. 2020;74:936-49.

15. Zhao Y, Huang Z, Huang J, Zhang C, Meng F. Phylogenetic analysis and expression differences of eye-related genes in cavefish genus Sinocyclocheilus. Integr Zool. 2020. https://doi.org/10.1111/1749-4877.12466.

16. Borowsky R. Breeding Astyanax mexicanus through natural spawning. Cold Spring Harb Protoc. 2008;2008:pdb-prot5091.

17. Lan J, Gan X, Wu T, Yang J. Cave fishes of Guangxi, China. 2013.

18. Huang $Q$, Cai $Y$, Xing X. Rocky desertification, antidesertification, and sustainable development in the karst mountain region of Southwest China. Ambio. 2008;37:390-2.

19. Jiang W-S, Li J, Lei X-Z, Wen Z-R, Han Y-Z, Yang J-X, et al. Sinocyclocheilus sanxiaensis, a new blind fish from the Three Gorges of Yangtze River provides insights into speciation of Chinese cavefish. Zool Res. 2019;40:552.

20. Zhao Y, Zhang C. Endemic fishes of Sinocyclocheilus (Cypriniformes: Cyprinidae) in China-species diversity, cave adaptation, systematics and zoogeography. Beijing: Science Press; 2009.

21. Romero A, Zhao Y, Chen X. The hypogean fishes of China. In: Chinese fishes. Dordrecht: Springer; 2009. p. 211-78.

22. Ma L, Zhao Y, Yang J. Cavefish of China. In: Encyclopedia of caves. Amsterdam: Elsevier; 2019. p. 237-54

23. Li J, Li XH, Mayden RL. Sinocyclocheilus brevifinus (Teleostei: Cyprinidae), a new species of cavefish from Guangxi, China. Zootaxa. 2014;3873:37-48.

24. Zhou J, Liu Q, Wang H, Yang L, Zhao D, Zhang T, et al. Description on a new species of Sinocyclocheilus in Guizhou. Sichuan J Zool. 2011;28:387-9.

25. Zhu D, Zhu Y. A new species of the genus Sinocyclocheilus (Cypriniformes, Cyprinidae) from Guangxi, China. Acta Zootaxonomica Sin. 2012;37:222-6

26. Zheng H, Xiu L, Yang J. A new species of Barbine genus Sinocyclocheilus (Teleostei: Cyprinidae) from Zuojiang river drainage in Guangxi, China. Environ Biol Fish. 2013;96:747-51.

27. Li J, Li X-H. Sinocyclocheilus gracilis, a new species of hypogean fish from Guangxi, South China (Teleostei: Cypriniformes: Cyprinidae). Ichthyol Explor Freshw. 2014;24:249-56.

28. Wang D, Zhao YH, Yang JX, Zhang CG. A new cavefish species from Southwest China, Sinocyclocheilus gracilicaudatus sp. nov. (Teleostei: Cypriniformes: Cyprinidae). Zootaxa. 2014;3768:583-90.

29. Chen Y-Q, Peng C-L, Zhang E. Sinocyclocheilus guanyangensis, a new species of cavefish from the Li-Jiang basin of Guangxi, China (Teleostei: Cyprinidae). Ichthyol Explor Freshw. 2016;27:1-8.

30. Luo F, Huang J, Liu X, Luo T, Wen Y. Sinocyclocheilus ronganensis Luo, Huang et Wen sp. nov., a new species belonging to Sinocyclocheilus Fang from Guangxi (Cypriniformes: Cyprinidae). J South Agric. 2016:47:650-5. 
31. Yang H, Li C, Chen Y, Li W. A new species of Sinocyclocheilus from Yunnan: Sinocyclocheilus wenshanensis. J Yunnan Univ Nat Sci Ed. 2017;39:507-12. https://doi.org/10.7540/j.ynu.20160534.

32. Zhou J, Li X, Hou X, Sun Z, Gao L, Zhao T. A new species of Sinocyclocheilus in Guizhou, China. Sichuan J Zool. 2009;28:321-3.

33. Wu T, Liao Z, Gan X, Li W. Two new species of Sinocyclocheilus in Guangxi, China (Cypriniefrmes, Cyprinidae). J Guangxi Norm Univ Nat Sci Ed. 2010;28:116-20.

34. Zhu D-G, Zhu Y, Lan J-H. Description of a new species of Barbinae, Sinocyclocheilus huangtianensis from China (Teleostei: Cypinidae). Dongwuxue Yanjiu. 2011;32(2):204-7.

35. Gan X, Wu T-J, Wei M-L, Yang J. A new blind barbine species, Sinocyclocheilus anshuiensis sp. nov. (Cypriniformes: Cyprinidae) from Guangxi, China. Dongwuxue Yanjiu. 2013;34(5):459-63.

36. Pan X-F, Li L, Yang J-X, Chen X-Y. Sinocyclocheilus xichouensis, a new species of golden-line fish from the Red River drainage in Yunnan, China (Teleostei: Cypriniformes). Dong wu xue yan jiu. 2013;34(4):368-73.

37. Cheng C, Pan X, Chen X, Li J, Ma L, Yang J. A new species of the genus Sinocyclocheilus (Teleostei: Cypriniformes), from Jinshajiang Drainage, Yunnan, China. Cave Res. 2015:1(2):1-4.

38. Lan Y, Qin X, Lan J, Xiu L, Yang J. A new species of the genus Sinocyclocheilus (Cypriniformes, Cyprinidae) from Guangxi, China. J Xinyang Norm Univ (Nat Sci Ed). 2017;30:97. https://doi.org/10.3969/j. issn.1003-0972.2017.01.021.

39. Li G, Wu J, Leng Y, Zhou R, Pan X, Han F, et al. A new species Sinocyclocheilus longshanensis sp. nov. from Yunnan. Chin Agric Sci Bull. 2018;34:153-8. https://doi.org/10.11924/j.issn.1000-6850.casb17080066.

40. Xiao H, Chen S, Liu Z, Zhang R, Li W, Zan R, et al. Molecular phylogeny of Sinocyclocheilus (Cypriniformes: Cyprinidae) inferred from mitochondrial DNA sequences. Mol Phylogenet Evol. 2005;36:67-77.

41. Jiang Y, Fu J, Zhang D, Zhao Y. Investigation on the lateral line systems of two cavefish: Sinocyclocheilus macrophthalmus and S. microphthalmus (Cypriniformes: Cyprinidae). J Bionic Eng. 2016;13:108-14.

42. Li P, Yang J. Complete mitochondrial genome and phylogenetic analysis of Sinocyclocheilus microphthalmus (Cypriniformes: Cyprinidae). Mitochondrial DNA Part B. 2019:4:3094-5.

43. Ardila A. The evolutionary concept of "preadaptation" applied to cognitive neurosciences. Front Neurosci. 2016;10:103.

44. Espinasa L, Borowsky R. Eyed cave fish in a karst window. J Cave Karst Stud. 2000;62:180-3.

45. Culver DC, Pipan T. The biology of caves and other subterranean habitats. Oxford: Oxford University Press; 2019

46. Trajano E, Bichuette ME, Kapoor BG. Biology of subterranean fishes. Boca Raton: CRC Press; 2010

47. He Y, Chen X-Y, Xiao T-Q, Yang J-X. Three-dimensional morphology of the Sinocyclocheilus hyalinus (Cypriniformes: Cyprinidae) horn based on synchrotron X-ray microtomography. Dong wu xue yan jiu. 2013;34(E4-5):E128-34.

48. Soares D, Pluviose M, Zhao Y. Ontogenetic development of the horn and hump of the Chinese cavefish Sinocyclocheilus furcodorsalis (Cypriniformes: Cyprinidae). Environ Biol Fish. 2019;102:741-6.

49. Gallo ND, Jeffery WR. Evolution of space dependent growth in the teleost Astyanax mexicanus. PLoS ONE. 2012;7:e41443.

50. Jeffery WR. Regressive evolution in Astyanax cavefish. Annu Rev Genet. 2009:43:25-47.

51. Protas M, Tabansky I, Conrad M, Gross JB, Vidal O, Tabin CJ, et al. Multi-trait evolution in a cave fish Astyanax mexicanus. Evol Dev. 2008;10:196-209.

52. Bradic M, Teotónio H, Borowsky RL. The population genomics of repeated evolution in the blind cavefish Astyanax mexicanus. Mol Biol Evol. 2013:30:2383-400.

53. Casane D, Rétaux S. Evolutionary genetics of the cavefish Astyanax mexicanus. Adv Genet. 2016:95:117-59.

54. Gross JB. The complex origin of Astyanax cavefish. BMC Evol Biol. 2012;12:1-12

55. Hubbs CL, Innes WT. The first known blind fish of the family Characidae: a new genus from Mexico. Occas Pap Mus Zool Univ Mich. 1936:342:1-7.

56. Gross JB, Furterer A, Carlson BM, Stahl BA. An integrated transcriptomewide analysis of cave and surface dwelling Astyanax mexicanus. PLoS ONE. 2013;8:e55659.

57. Carlson BM, Onusko SW, Gross JB. A high-density linkage map for Astyanax mexicanus using genotyping-by-sequencing technology. G3 Genes Genomes Genet. 2015;5:241-51.
58. Powers AK, Davis EM, Kaplan SA, Gross JB. Cranial asymmetry arises later in the life history of the blind Mexican cavefish, Astyanax mexicanus. PLoS ONE. 2017:12:e0177419.

59. Herman A, Brandvain Y, Weagley J, Jeffery WR, Keene AC, Kono TJY, et al. The role of gene flow in rapid and repeated evolution of cave-related traits in Mexican tetra, Astyanax mexicanus. Mol Ecol. 2018;27:4397-416.

60. Bradic M, Beerli P, García-de León FJ, Esquivel-Bobadilla S, Borowsky RL. Gene flow and population structure in the Mexican blind cavefish complex (Astyanax mexicanus). BMC Evol Biol. 2012;12:9.

61. Wilkens $H$, Strecker U. Surface and cave populations of Mexican Astyanax. In: Evolution in the dark. Berlin: Springer; 2017. p. 37-52.

62. Krishnan J, Rohner N. Cavefish and the basis for eye loss. Philos Trans R Soc B. 2017:372:20150487.

63. Wilkens H. Genetics and hybridization in surface and cave Astyanax (Teleostei): a comparison of regressive and constructive traits. Biol J Lin Soc. 2016;118:911-28.

64. Staden R. The Staden sequence analysis package. Mol Biotechnol. 1996:5:233-41.

65. Thompson JD, Gibson TJ, Higgins DG. Multiple sequence alignment using ClustalW and ClustalX. Curr Protoc Bioinf. 2003. https://doi. org/10.1002/0471250953.bi0203s00.

66. Tamura K, Stecher G, Peterson D, Filipski A, Kumar S. MEGA6: molecular evolutionary genetics analysis version 6.0. Mol Biol Evol. 2013;30:2725-9.

67. Santorum JM, Darriba D, Taboada GL, Posada D. Jmodeltest.org: selection of nucleotide substitution models on the cloud. Bioinformatics. 2014;30:1310-1.

68. Suchard MA, Lemey P, Baele G, Ayres DL, Drummond AJ, Rambaut A. Bayesian phylogenetic and phylodynamic data integration using BEAST 1.10. Virus evolution. 2018:4:vey016.

69. Miller MA, Pfeiffer W, Schwartz T. The CIPRES science gateway: a community resource for phylogenetic analyses. In: Proceedings of the 2011 TeraGrid conference on extreme digital discovery - TG'11. Salt Lake City, Utah: ACM Press; 2011. p. 1. https://doi.org/10.1145/2016741.2016785.

70. Rambaut A, Drummond AJ, Xie D, Baele G, Suchard MA. Posterior summarization in Bayesian phylogenetics using Tracer 1.7. Syst Biol. 2018;67:901.

71. Drummond AJ, Suchard MA, Xie D, Rambaut A. Bayesian phylogenetics with BEAUti and the BEAST 1.7. Mol Biol Evol. 2012;29:1969-73.

72. RohlfF. tpsDig, ver 2.16: department of ecology and evolution. 2010.

73. Rohlf FJ. tpsUtil, file utility program, version 1.26. Department of Ecology and Evolution, State University of New York at Stony Brook. 2004.

74. Gunz P, Mitteroecker P. Semilandmarks: a method for quantifying curves and surfaces. Hystrix. 2013:24:103-9.

75. Adams DC, Otárola-Castillo E. geomorph: an R package for the collection and analysis of geometric morphometric shape data. Methods Ecol Evol. 2013:4:393-9.

76. Adams DC, Rohlf FJ, Slice DE. A field comes of age: geometric morphometrics in the 21st century. Hystrix. 2013;24:7.

77. Nielsen R. Mapping mutations on phylogenies. Syst Biol. 2002;51:729-39.

78. Huelsenbeck JP, Nielsen R, Bollback JP. Stochastic mapping of morphological characters. Syst Biol. 2003;52:131-58.

79. Evans ME, Smith SA, Flynn RS, Donoghue MJ. Climate, niche evolution, and diversification of the "bird-cage" evening primroses (Oenothera, sections Anogra and Kleinia). Am Nat. 2009:173:225-40.

80. Revell LJ. phytools: an R package for phylogenetic comparative biology (and other things). Methods Ecol Evol. 2012;3:217-23.

81. Sidlauskas B. Continuous and arrested morphological diversification in sister clades of characiform fishes: a phylomorphospace approach. Evolution. 2008;62:3135-56.

82. Team RC. R: A language and environment for statistical computing. 2013.

83. Parks DH, Mankowski T, Zangooei S, Porter MS, Armanini DG, Baird DJ, et al. GenGIS 2: geospatial analysis of traditional and genetic biodiversity, with new gradient algorithms and an extensible plugin framework. PLoS ONE. 2013;8:e69885.

\section{Publisher's Note}

Springer Nature remains neutral with regard to jurisdictional claims in published maps and institutional affiliations. 